\title{
Variants in the CYP7B1 gene region do not affect natural resistance to HIV-1 infection
}

\author{
Manuela Sironi ${ }^{1 \dagger}$, Mara Biasin ${ }^{2^{*}+}$, Chiara Pontremoli ${ }^{1}$, Rachele Cagliani ${ }^{1}$, Irma Saulle ${ }^{2}$, Daria Trabattoni ${ }^{2}$, \\ Francesca Vichi ${ }^{3}$, Sergio Lo Caputo ${ }^{3}$, Francesco Mazzotta ${ }^{3}$, Wbeimar Aguilar-Jimenez ${ }^{4}$, Maria Teresa Rugeles ${ }^{4}$, \\ Samandhy Cedeno ${ }^{5,6}$, Jorge Sanchez ${ }^{7}$, Christian Brander ${ }^{5,6}$ and Mario Clerici $i^{8,9}$
}

\begin{abstract}
Background: The genetic bases of natural resistance to HIV-1 infection remain largely unknown. Recently, two genome-wide association studies suggested a role for variants within or in the vicinity of the CYP7B1 gene in modulating HIV susceptibility. CYP7B1 is an appealing candidate for this due to its contribution to antiviral immune responses. We analyzed the frequency of two previously described CYP7B1 variants (rs6996198 and rs10808739) in three independent cohorts of HIV-1 infected subjects and HIV-1 exposed seronegative individuals (HESN).

Findings: rs6996198 and rs10808739 were genotyped in three case/control cohorts of sexually-exposed HESN and HIV-1-infected individuals from Italy, Peru and Colombia. Comparison of the allele and genotype frequencies of the two SNPs under different models showed that the only significant difference was seen for rs6996198 in the Peruvian sample (nominal $p=0.048$, dominant model). For this variant, a random-effect meta-analysis yielded non-significant results (dominant model, $p=0.78$ ) and revealed substantial heterogeneity among cohorts. No significant effect of the rs10808739 allelic status on HIV-1 infection susceptibility (additive model, $p=0.30$ ) emerged from the meta-analysis.

Conclusions: Although our study had limited power to detect association due to the small sample size, comparisons among the three cohorts revealed very similar allelic and genotypic frequencies in HESN and HIV-1 positive subjects. Overall, these data indicate that the two GWAS-defined variants in the CYP7B1 region do not strongly influence HIV-1 infection susceptibility.
\end{abstract}

Keywords: HIV, HESN, CYP7B1, Resistance to infection

\section{Findings}

Individual variation in susceptibility to HIV-1 infection is well documented [1-3], and a minority of individuals (15\% of the HIV exposed people), usually referred to as HIV-1 exposed seronegative individuals (HESN), does not become productively infected despite multiple exposures to the virus $[2,4,5]$. Because only a minority of these subjects is homozygous for the CCR $5 \Delta 32$ deletion [6], it is logical to postulate that other, additional genetic factors can modulate susceptibility to HIV-1 infection. Some of such factors were identified through

\footnotetext{
*Correspondence: mara.biasin@unimi.it

${ }^{\dagger}$ Manuela Sironi and Mara Biasin contributed equally to this work

2 Department of Biomedical and Clinical Sciences "L. Sacco", University

of Milan, Via G.B. Grassi 74, 20157 Milan, Italy

Full list of author information is available at the end of the article
}

candidate gene approaches; only few of them however were replicated across different samples [6]. In recent years, genome-wide association studies (GWAS) have been used for the identification of common variants that underlie complex phenotypes. Among these, two GWASs for HIV-1 infection susceptibility have suggested a role of variants within or in the vicinity of the CYP7B1 gene in this phenomenon [7, 8]. In one study, HIV-1 infected individuals were compared with HIV-1 negative subjects [7]. Using meta-analysis of two European cohorts and further validation in European Americans, a single signal with genome-wide significance was detected at rs6996198, a SNP that is located downstream the CYP7B1 gene and possibly modulates its expression [7]. Another variant in CYP7B1 (rs10808739, in intron 1) was described in a GWAS of HIV-1 serodiscordant couples 
from Eastern and Southern Africa. Although the variant did not reach genome-wide significance, it was one of the top signals in the study [8].

The CYP7B1 gene encodes an enzyme belonging to the cytochrome P450 superfamily that is involved in cholesterol catabolism by inactivation of oxysterols and their subsequent conversion into bile salts, mainly in the liver, brain, and reproductive tract [9]. Controlling intracellular cholesterol metabolism is essential for the correct entry, assembly and budding of virions, and many viruses are dependent on cellular lipid metabolisms to ensure the correct budding of functional virions from infected cells [10]. HIV alters cholesterol trafficking switching it from a physiological efflux to a virus-controlled transport, thus reducing the ability of a cell to export cholesterol. CYP7B1 also modulates several immune functions, as well including proinflammatory cytokine release [11] and, via its catabolite 25-hydroxycholesterol [12], programmed cell death [13] and the synthesis of IgA [14], a class of antibodies that has been associated with resistance to HIV infection [15]. Hence, CYP7B1 represents an appealing candidate as a modifier of infection susceptibility due to its contribution to the synthesis of virions and the initiation of antiviral defense.

To verify the role played by CYP7B1 in resistance to HIV infection we recruited 125 Italian HESN exposed through unprotected sexual intercourse (SexExp-HESN). Inclusion criteria were a history of multiple unprotected sexual episodes for more than 4 years at the time of enrolment, with at least three episodes of at-risk intercourse within 4 months prior to study entry and an average of 30 (range, 18 to $>100$ ) reported unprotected sexual contacts per year [5]. Infection in HESN was ruled-out by plasma HIV RNA and proviral DNA analyses. HESN and 114 seropositive (SP) partners were recruited at the S. M. Annunziata Hospital, Florence; all of them were Italian of Caucasian origin.

Sixty-two Colombian SexExp-HESN and 51 SP partners were also included. The inclusion criteria for these HESN subjects were previously reported [16] and included a negative HIV-1/2 ELISA test within 1 month of sample taking. The similar ancestry component and pair-wise fixation index (FST) values in the Colombian cohort [17] indicated no intra-cohort stratification by ethnicity.

The third HESN cohort was recruited in Peru and has been described $[18,19]$ both in terms of host genetics (HLA and KIR) and immune reactivity to HIV and viral co-pathogens. The similar frequency of HLA and KIR alleles [18] suggested no major intra-cohort stratification. For this study, samples were available for a total of 130 HESN and 95 SP individuals, all of whom were recruited at IMPACTA clinics across Lima. HESN were tested on a 3-monthly basis for newly acquired HIV infection. Risk criteria for the HESN cohort were more than five different sexual partners over the last 3 months, reported STI over the last 6 months, sexual intercourse with a known HIV SP partner in the last 6 months and having accepted money for sex as described previously [J. Coll et al. Bangkok AIDS Vaccine meeting 2011, Factors influencing recruitment, retention and seroconversion rates in MSM at high risk for HIV infection in Lima and Barcelona].

The study was designed and performed according to the Helsinki declaration (1975 revised in 2000) and was approved by the Ethics Committee of the participating units. All subjects provided written informed consent to participate in this study.

Genotyping of CYP7B1 rs6996198 and rs10808739 was performed by TaqMan probe assays (TaqMan SNP genotyping assay, Applied Biosystems, Foster City, CA, USA) using the allelic discrimination real-time PCR method. Genotyping rate was $>0.90$ for both SNPs in all samples. The two SNPs complied to Hardy-Weinberg equilibrium in all samples with the only exception of rs6966198 in the SP Peruvian sample (uncorrected $\mathrm{p}$ value $=0.02$ ). Association analysis for single variants was performed using logistic regressions under different models using the PLINK software [20]. Meta-analysis was performed using a random effect model using the $\mathrm{R}$ package "meta" ( $\mathrm{R}$ package version 4.3-0. http://CRAN.R-project.org/ package $=$ meta). Linkage analysis was performed using HaploReg v2 [21].

The two CYP7B1 SNPs previously suggested to be associated with HIV-1 infection susceptibility (rs6996198 and rs10808739) are located $\sim 177 \mathrm{~kb}$ apart and are not in strong linkage disequilibrium (LD) in individuals of European or American descent $\left(r^{2}=0.15\right.$ and $r^{2}=0.12$, respectively, http://analysistools.nci.nih.gov/LDlink/). The two variants were genotyped in three distinct cohorts of HESN and geographically matched HIV-1 SP subjects (Table 1). The main risk factor in all HESN was unprotected sex.

The allele and genotype frequencies at the two SNPs were compared under different models. The only significant difference was observed for rs6996198 in the Peruvian sample (nominal $\mathrm{p}=0.048$, dominant model): as in the original GWAS [7], the frequency of TT + CT genotypes was lower in SP compared to HESN subjects (Table 2). For this variant, a random-effect metaanalysis yielded non-significant results (dominant model, $\mathrm{p}=0.78$, OR $=1.0971,95 \%$ CI: 0.5727-2.1016) and revealed substantial heterogeneity among cohorts (Cochrane's Q p value $=0.047, \mathrm{I}^{2}=67.32$ ). Likewise, meta-analysis indicated no significant effect of rs10808739 allelic status on HIV-1 infection susceptibility (additive model, $\mathrm{p}=0.30, \mathrm{OR}=0.85,95 \% \mathrm{CI}$ : 
Table 1 Demographic status of the populations examined

\begin{tabular}{|c|c|c|c|c|c|c|}
\hline \multirow[t]{2}{*}{ Characteristics } & \multicolumn{2}{|l|}{ Colombia } & \multicolumn{2}{|l|}{ Italy } & \multicolumn{2}{|l|}{ Peru } \\
\hline & $S P(n=51)$ & $\operatorname{HESN}(n=62)$ & $S P(n=114)$ & $\operatorname{HESN}(n=125)$ & $S P(n=95)$ & $\operatorname{HESN}(n=130)$ \\
\hline Age, mean years $\pm S D$ & $33.9 \pm 7.5$ & $35.1 \pm 10.6$ & $42.4 \pm 8.8$ & $41.6 \pm 9.1$ & $30.8 \pm 6.7$ & $31.2 \pm 10.7$ \\
\hline Males, n (\%) & $26(50)$ & $27(44.2)$ & $69(60.5)$ & $53(42.4)$ & $94(99)$ & $123(90)$ \\
\hline $\begin{array}{l}\text { Viral load, median } \\
\text { copies/mL (inter- } \\
\text { quartile range) }\end{array}$ & $2569(488-25,075)$ & - & $10,950(395-27,410)$ & - & $\begin{array}{l}29,694(11,162- \\
63,381)\end{array}$ & - \\
\hline $\begin{array}{l}\text { CD4+ T cell/ } \mu \mathrm{L} \text { count, } \\
\text { median (interquar- } \\
\text { tile range) }\end{array}$ & $366(190-568)$ & - & $369(239-554)$ & - & $417^{\mathrm{e}}(331-544)$ & - \\
\hline $\begin{array}{l}\text { Monthly unprotected } \\
\text { sexual episodes, } \\
\text { mean (range) }\end{array}$ & $8(1-30)$ & $8(1-30)$ & $3(1.5-10)$ & & $7(1-25)$ & \\
\hline $\begin{array}{l}\text { Previous history of } \\
\text { sexually transmit- } \\
\text { ted diseases and/ } \\
\text { or AIDS-defining } \\
\text { illnesses (\%) }\end{array}$ & 40 & $22^{\mathrm{a}}$ & 39 & - & $n d^{d}$ & 29 \\
\hline $\begin{array}{l}\text { Heterosexual orienta- } \\
\text { tion (\%) }\end{array}$ & 79 & 90 & 100 & 100 & $n d^{d}$ & 17 \\
\hline $\begin{array}{l}\text { Homosexual orienta- } \\
\text { tion (\%) }\end{array}$ & 3 & 2 & 0 & 0 & $n d^{d}$ & 44 \\
\hline $\begin{array}{l}\text { Bisexual orientation } \\
\quad(\%)\end{array}$ & 17 & 7 & 0 & 0 & $n d^{d}$ & 39 \\
\hline \multirow[t]{3}{*}{ Ethnicity, ancestry ${ }^{\mathrm{b}}(\%)$} & Afr: 25 & Afr: 22 & \multirow{3}{*}{$\begin{array}{l}\text { European (Tuscan): } \\
100\end{array}$} & \multirow{3}{*}{$\begin{array}{l}\text { European (Tuscan): } \\
\quad 100\end{array}$} & \multirow[t]{3}{*}{ Mestizo: 100} & Mestizo: 89 \\
\hline & Amer: 40 & Amer: 42 & & & & Indigenous: 5 \\
\hline & Eur: 34 & Eur: 35 & & & & Others: 6 \\
\hline
\end{tabular}

SP seropositives, HESN HIV-1 exposed seronegative, SD standard deviation, yrs years, Afr African, Amer Amerindian, Eur European

a In HESNs sexually transmitted diseases but no AIDS-defining illnesses

b Ancestry of the Colombian cohort was previously reported [17]

c In Peru, this refers to number of partners, not sexual episodes

d "nd" is not determined. The majority is gay men but this question was not formally asked

e Cohort inclusion criteria was CD4 count >250 (requested by ethics board)

0.6309-1.1531); no heterogeneity was observed for this variant (Cochrane's Q p value $=0.700, \mathrm{I}^{2}=0$ ).

The original study that reported association of rs6996198 with protection from HIV-1 acquisition was performed in European populations; analysis of the 1000 Genomes Project data (Phase I) indicated that no variant in strong $\operatorname{LD}\left(r^{2} \geq 0.8\right)$ with rs6996198 segregates in Europeans (nor in Americans). As for rs10808739, it was initially described in cohorts of African ancestry. LD analysis in African populations indicated that rs10808739 is in strong $\mathrm{LD}\left(r^{2} \geq 0.8\right)$ with several variants, the majority of which show a similar LD pattern in Europeans and Americans (Fig. 1). Thus, it seems unlikely that the negative results we obtained are due to substantially different haplotype structures across populations.

CYP7B1 plays a major role in the synthesis of HIV and in immunity and is thus a likely candidate in the modulation of susceptibility to HIV. Results, however, did not confirm the previously reported associations, suggesting that these particular $C Y P 7 B 1$ genetic variants do not play a role in HIV-1 infection susceptibility, and indicating that other variants will need to be analyzed. A limitation of our study is the relatively small sample sizes of the HESN and HIV-1 infected cohorts, resulting in low power to detect potentially existing associations. Nonetheless, the combined sample size was comparable to that analyzed in the serodiscordant couple study that detected an association at rs10808739 [8]. As for rs6966198, it reached genome-wide significance in a relatively large two-stage study that employed a different design from the one applied herein [7]. Thus, in that case, HIV-1-infected individuals were compared to HIV-1 negative subjects, irrespective of their exposure status. This may have determined a proportion of $\mathrm{HIV}-1$ negative controls to be misclassified-i.e. most of with a consequent reduction of power. Our analyses were performed in well characterized HESN cohorts hence overcoming this problem; a degree of misclassification may nevertheless still remain for HIV-1 positive subjects because their exposure history may not always be properly and completely recorded 
Table 2 Association of rs6966198 and rs10808739 with HIV-1 infection susceptibility

\begin{tabular}{|c|c|c|c|c|c|c|c|c|c|}
\hline \multirow[t]{2}{*}{ Sample } & \multicolumn{4}{|c|}{ Genotype frequency } & \multicolumn{2}{|c|}{ Allelic frequency } & \multirow{2}{*}{$p_{\text {additive }}^{\mathbf{b}}$} & \multirow{2}{*}{$p_{\text {dominant }}^{c}$} & \multirow[t]{2}{*}{$p_{\text {recessive }}^{\mathrm{d}}$} \\
\hline & $\mathrm{CC}$ & $\mathrm{CT}$ & TT & $\mathrm{CT}+\mathrm{TT}^{\mathrm{a}}$ & $C$ & $\mathbf{T}$ & & & \\
\hline \multicolumn{10}{|l|}{ rs6966198 } \\
\hline Italian HESN & 0.648 & 0.304 & 0.048 & 0.35 & 0.8 & 0.2 & 0.112 & 0.137 & 0.373 \\
\hline Italian SP & 0.551 & 0.373 & 0.076 & 0.45 & 0.737 & 0.263 & & & \\
\hline Colombian HESN & 0.661 & 0.274 & 0.065 & 0.339 & 0.798 & 0.202 & 0.557 & 0.83 & 0.309 \\
\hline Colombian SP & 0.681 & 0.298 & 0.021 & 0.319 & 0.83 & 0.17 & & & \\
\hline Peruvian HESN & 0.685 & 0.292 & 0.023 & 0.315 & 0.831 & 0.169 & 0.147 & 0.048 & 0.399 \\
\hline Peruvian SP & 0.805 & 0.152 & 0.043 & 0.196 & 0.88 & 0.12 & & & \\
\hline \multirow[t]{2}{*}{ Sample } & \multicolumn{4}{|c|}{ Genotype frequency } & \multicolumn{2}{|c|}{ Allelic frequency } & $p_{\text {additive }}^{\mathbf{b}}$ & $p_{\text {dominant }}^{c}$ & $p_{\text {recessive }}^{\mathrm{d}}$ \\
\hline & GG & GA & AA & - & $\mathbf{G}$ & A & & & \\
\hline \multicolumn{10}{|l|}{ rs10808739 } \\
\hline Italian HESN & 0.58 & 0.33 & 0.09 & - & 0.746 & 0.254 & 0.392 & 0.267 & 0.961 \\
\hline Italian SP & 0.5 & 0.412 & 0.088 & - & 0.706 & 0.294 & & & \\
\hline Colombian HESN & 0.71 & 0.226 & 0.064 & - & 0.823 & 0.177 & 0.831 & 0.787 & 0.275 \\
\hline Colombian SP & 0.686 & 0.294 & 0.02 & - & 0.833 & 0.167 & & & \\
\hline Peruvian HESN & 0.794 & 0.198 & 0.008 & - & 0.893 & 0.107 & 0.302 & 0.38 & 0.389 \\
\hline Peruvian SP & 0.741 & 0.235 & 0.024 & - & 0.859 & 0.141 & & & \\
\hline \multicolumn{10}{|c|}{ a Genotypic counts (dominant model, as in [7]) } \\
\hline \multicolumn{10}{|c|}{ b Logistic regression $\mathrm{p}$ value for an additive model } \\
\hline \multicolumn{10}{|c|}{${ }^{c}$ Logistic regression $\mathrm{p}$ value for a dominant model } \\
\hline${ }^{d}$ Logistic regression & le for a re & ve model & & & & & & & \\
\hline
\end{tabular}

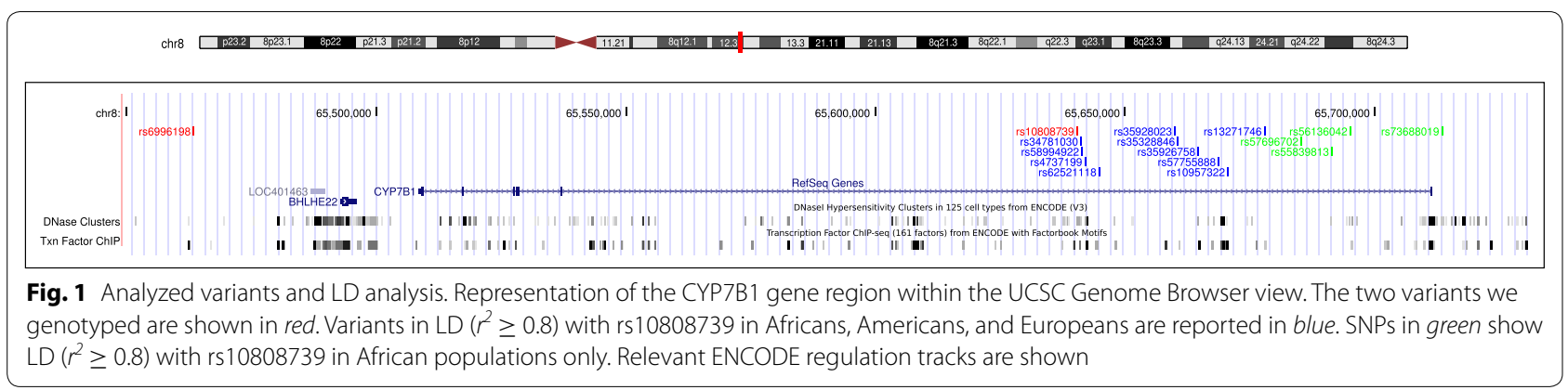

and their group is likely to include individuals in a wide susceptibility range.

Taking into account these limitations, comparisons in the three cohorts showed very similar allelic and genotypic frequencies in HESN and HIV-1-infected individuals, with the only exception being the Peruvian sample that reached nominal significance for rs6996198. For the same variant, the Italian cohort also showed some difference in frequency, albeit not significant, that was in the opposite direction to that reported by the original association study [7] and to that observed in Peruvians. The original study that identified rs6996198 as potentially associated with HIV-1 infection susceptibility estimated an OR of 0.64 with narrow confidence intervals (95\%
IC: 0.54-075). Herein, also due to heterogeneity among samples, we obtained an OR very close to 1 with wide uncertainty (95\% IC: $0.5727-2.1016$ ). Thus, our results do not imply that rs6996198 has no role in modulating HIV-1 infection susceptibility, but indicate that its effect (if any) is small. Overall, these data lead us to conclude that the two genotyped variants in the CYP7B1 region do not strongly influence HIV-1 infection susceptibility.

\section{Authors' contributions}

$M S, M B$ and MC, conceived the study and wrote the paper; IS, RC and CP performed the experiments and analyzed the data; SLC, FM, FV and DT enrolled and selected the cohort of HESN subjects in Florence; WA-J, and MTR enrolled and selected the cohort of HESN subjects in Colombia; CB enrolled and selected the cohort of HESN subjects in Peru. All authors read and approved the final manuscript. 


\section{Author details}

${ }^{1}$ Scientific Institute IRCCS E. MEDEA, Bioinformatics, 23842 Bosisio Parini, Italy. ${ }^{2}$ Department of Biomedical and Clinical Sciences "L. Sacco", University of Milan, Via G.B. Grassi 74, 20157 Milan, Italy. ${ }^{3}$ S. Maria Annunziata Hospital, 50122 Florence, Italy. ${ }^{4}$ Immunovirology Group, School of Medicine, University of Antioquia UdeA, Medellín, Colombia. ${ }^{5}$ AIDS Research Institute-IrsiCaixa-HIVACAT, Hospital Germans Trias i Pujol, Universitat Autònoma de BarceIona, Badalona, Institució Catalana de Recerca i Estudis Avançats (ICREA), Barcelona, Spain. ${ }^{6}$ University of Vic and Central Catalonia, Vic, Spain. ${ }^{7}$ Asociación Civil Impacta Salud y Educación, Lima, Peru. ${ }^{8}$ Department of Physiopathology and Transplantation, University of Milan, 20090 Milan, Italy. ${ }^{9}$ Don C. Gnocchi Foundation ONLUS, IRCCS, 20148 Milan, Italy.

\section{Acknowledgements}

This work was supported by: Estrategia de Sostenibilidad 2014-2015 de la Universidad de Antioquia; NIH-NIDCR R01 DE018925-04; the HIVACAT program and CUTHIVAC 241904. Thanks to "Grupo de Vida" foundation in MedellinColombia, to the staff of HERES Health, from Santa Marta-Colombia for their collaboration in patient recruitment.

\section{Compliance with ethical guidelines}

\section{Competing interests}

The authors declare that they have no competing interests.

Received: 21 July 2015 Accepted: 14 September 2015

Published online: 24 September 2015

\section{References}

1. Plummer FA, Ball TB, Kimani J, Fowke KR. Resistance to HIV-1 infection among highly exposed sex workers in Nairobi: what mediates protection and why does it develop? Immunol Lett. 1999;66(1-3):27-34.

2. Fowke KR, Nagelkerke NJ, Kimani J, Simonsen JN, Anzala AO, Bwayo JJ, et al. Resistance to HIV-1 infection among persistently seronegative prostitutes in Nairobi, Kenya. Lancet. 1996:348(9038):1347-51.

3. Piacentini L, Biasin M, Fenizia C, Clerici M. Genetic correlates of protection against HIV infection: the ally within. J Intern Med. 2009;265(1):110-24

4. Clerici M, Giorgi JV, Chou CC, Gudeman VK, Zack JA, Gupta P, et al. Cell mediated immune response to human immunodeficiency virus type 1 (HIV-1) in seronegative homosexuals with recent sexual exposure to HIV1. JID. 1992;165:1012-9.

5. Miyazawa M, Lopalco L, Mazzotta F, Lo Caputo S, Veas F, Clerici M. The, "immunologic advantage" of HIV-exposed seronegative individuals. AIDS. 2009;23:161-76.

6. McLaren PJ, Fellay J. Human genetic variation in HIV disease: beyond genome-wide association studies. Curr Opin HIV AIDS. 2015:10(2):110-5.

7. Limou S, Delaneau O, van Manen D, An P, Sezgin E, Le Clerc S, et al. Multicohort genomewide association study reveals a new signal of protection against HIV-1 acquisition. J Infect Dis. 2012;205(7):1155-62.
8. Lingappa JR, Petrovski S, Kahle E, Fellay J, Shianna K, McElrath MJ, et al. Genomewide association study for determinants of HIV-1 acquisition and viral set point in HIV-1 serodiscordant couples with quantified virus exposure. PLoS One. 2011;6(12):e28632.

9. Yantsevich AV, Dichenko YV, Mackenzie F, Mukha DV, Baranovsky AV, Gilep AA, et al. Human steroid and oxysterol $7 \alpha$-hydroxylase CYP7B1: substrate specificity, azole binding and misfolding of clinically relevant mutants. FEBS J. 2014;281(6):1700-13.

10. Chukkapalli V, Heaton NS, Randall G. Lipids at the interface of virus-host interactions. Curr Opin Microbiol. 2012;15:512-8.

11. Dulos J, van der Vleuten MA, Kavelaars A, Heijnen CJ, Boots AM. CYP7B expression and activity in fibroblast-like synoviocytes from patients with rheumatoid arthritis: regulation by proinflammatory cytokines. Arthritis Rheum. 2005;52(3):770-8.

12. Martin C, Bean R, Rose K, Habib F, Seckl J. Cyp7b1 catalyses the 7alphahydroxylation of dehydroepiandrosterone and 25-hydroxycholesterol in rat prostate. Biochem J. 2001;355(Pt 2):509-15.

13. Ayala-Torres S, Moller PC, Johnson BH, Thompson EB. Characteristics of 25-hydroxycholesterol-induced apoptosis in the human leukemic cell line CEM. Exp Cell Res. 1997;235(1):35-47.

14. Bauman DR, Bitmansour AD, McDonald JG, Thompson BM, Liang G, Russell DW. 25-Hydroxycholesterol secreted by macrophages in response to Toll-like receptor activation suppresses immunoglobulin A production. Proc Natl Acad Sci USA. 2009;106(39):16764-9.

15. Broliden K, Hinkula J, Devito C, Kiama P, Kimani J, Trabbatoni D, et al. Functional HIV-1 specific IgA antibodies in HIV-1 exposed, persistently IgG seronegative female sex workers. Immunol Lett. 2001;79(1-2):29-36.

16. Zapata W, Rodriguez B, Weber J, Estrada H, Quiñones-Mateu ME, Zimermman PA, et al. Increased levels of human beta-defensins mRNA in sexually HIV-1 exposed but uninfected individuals. Curr HIV Res. 2008;6(6):531-8.

17. Zapata W, Aguilar-Jiménez W, Pineda-Trujillo N, Rojas W, Estrada H, Rugeles MT. Influence of CCR5 and CCR2 genetic variants in the resistance/ susceptibility to HIV in serodiscordant couples from Colombia. AIDS Res Hum Retroviruses. 2013;29(12):1594-603.

18. Olvera A, Pérez-Álvarez S, Ibarrondo J, Ganoza C, Lama JR, Lucchetti A, et al. The HLA-C*04: 01/KIR2DS4 gene combination and human leukocyte antigen alleles with high population frequency drive rate of HIV disease progression. AIDS. 2015;29(5):507-17.

19. Xu GJ, Kula T, Xu Q, Li MZ, Vernon SD, Ndung'u T, et al. Comprehensive serological profiling of human populations using a synthetic human virome. Science. 2015;348(6239):aaa0698.

20. Purcell S, Neale B, Todd-Brown K, Thomas L, Ferreira MA, Bender D, et al. PLINK: a tool set for whole-genome association and population-based linkage analyses. Am J Hum Genet. 2007;81:559-75.

21. Ward LD, Kellis M. HaploReg: a resource for exploring chromatin states, conservation, and regulatory motif alterations within sets of genetically linked variants. Nucleic Acids Res. 2012;40(Database issue):D930-4.

\section{Submit your next manuscript to BioMed Central and take full advantage of:}

- Convenient online submission

- Thorough peer review

- No space constraints or color figure charges

- Immediate publication on acceptance

- Inclusion in PubMed, CAS, Scopus and Google Scholar

- Research which is freely available for redistribution 